\title{
L-Band Multiwavelength Single-Longitudinal Mode Fiber Laser for Sensing Applications
}

\author{
R. A. Perez-Herrera, A. Ullan, D. Leandro, M. Fernandez-Vallejo, M. A. Quintela, A. Loayssa, Member, IEEE, \\ J. M. Lopez-Higuera, Senior Member, IEEE, and M. Lopez-Amo, Senior Member, IEEE
}

\begin{abstract}
In this work, a novel single-longitudinal-mode (SLM) four-wavelength laser configuration for sensing applications in the L-band is proposed and experimentally demonstrated. The sensor system presented here is based on ring resonators, and employs fiber Bragg gratings to select the operation wavelengths. The stable SLM operation is guaranteed when all the lasing channels present similar output powers. It is also experimentally demonstrated that when a SLM behavior is achieved, lower output power fluctuations are obtained. Characterization of the lasing structure for temperature sensing is also shown.
\end{abstract}

Index Terms-Erbium-doped fiber (EDF), fiber Bragg grating (FBG), multiwavelength laser, optical fiber amplifier, optical fiber ring laser, single-longitudinal mode (SLM).

\section{INTRODUCTION}

$\mathbf{T}$ HE utilization of fiber lasers for fiber optic sensors multiplexing has attracted much attention since the first demonstrations in the early 90's [1]. These lasers may be the optical source of a multiplexing network [2], or the laser itself may be the multiplexing structure [1], [3]. This last option offers high signal to noise ratios and it is particularly appealing for remote sensing applications [4]-[6].

Long-wavelength band (L-band) operation (from 1565 to $1625 \mathrm{~nm}$ ) in fiber optic sensing is limited by the higher price of the utilized optical components in comparison with the C-band (from 1530 to $1565 \mathrm{~nm}$ ) ones and because of a slightly higher attenuation in the optical fiber. However, this band is especially interesting for gas sensing applications, among others.

In addition to this, L-band EDFA is one of the key devices for the WDM transmission networks, which is combined with the conventional-wavelength-band (C-band) EDFA between 1525 and $1565 \mathrm{~nm}$ to produce very wide broadband amplification. The advantage of the utilization of L-band EDFAs is the reduction of four-wave mixing (FWM) problem in dispersion-shifted fibers (DSF), and it can also easily achieve flat gain even without a gain-flattening filter (GFF) rather than the C-band EDFA. Recently many researches are striving to develop L-band EDFAs.

Manuscript received June 14, 2011; revised September 21, 2011; accepted October 24, 2011. This work was supported in part by the Spanish Comisión Interministerial de Ciencia y Tecnología within project TEC2010-20224-C02.

R. A. Perez-Herrera, D. Leandro, M. Fernandez-Vallejo, A. Loayssa, and M. Lopez-Amo are with the Department of Electric and Electronic Engineering, Universidad Pública de Navarra, E-31006 Pamplona, Spain (e-mail: rosa.perez@unavarra.es; dani.leandro.glez@gmail.com; montserrat.fernandez@unavarra.es; alayn.loayssa@unavarra.es; mla@unavarra.es).

A. Ullan, M. A. Quintela, and J. M. Lopez-Higuera are with the Photonics Engineering Group, University of Cantabria, Cantabria, Spain (e-mail: angel. ullan@unican.es; quintelm@unican.es; higuera@teisa.unican.es).

Digital Object Identifier 10.1109/JLT.2011.2174138
To this goal, some aspects such as EDF length, pump configuration, pump wavelength, and with non-silica based EDF fibers are being explored [7].

L-band tunable lasers are also important for testing and measuring L-band devices for WDM transmission systems. The design of L-band EDFAs is essential for developing L-band tunable erbium-doped fiber lasers (EDFLs) [8], [9].

The advantage of extending the range of wavelength operation of fiber lasers into the L-band expands the limits of present day tunable sources and hence the detection of gases with absorption lines in the L-band [10]. In this region there are several gases of interest such us carbon dioxide $\left(\mathrm{CO}_{2}\right)$ and hydrogen sulphide $\left(\mathrm{H}_{2} \mathrm{~S}\right)[11]$.

Detection of these trace gases such as methane, carbon monoxide and carbon dioxide is extremely important for both pollution monitoring and safety reasons in the oil and gas industries, in water treatment plants, in landfill sites and in commercial or domestic environments, where methane gas may filter up through the ground and create an explosion hazard (the lower explosive limit, LEL, for methane is $5 \%$ by volume methane gas) [12]. Fiber optical sensors based on direct absorption spectroscopy, operating in the 1-2 $\mu \mathrm{m}$ spectral region, allow safe, remote location of sensors combined with the availability of low cost fiber components, connectors and compact gas cells. Several demonstrations of the application of the L-band hollow-core photonic bandgap fibers (HC-PBF) to the detection of methane have been reported in the literature [13]. It is therefore of commercial interest to develop portable gas sensors, based on optical techniques [14]. Optical sensors have the potential advantages of: (i) intrinsically safe, (ii) ability to detect a specific gas by selection of appropriate wavelengths, (iii) able to operate in zero-oxygen environment (e.g., for purging of pipe lines), and (iv) low cost of maintenance, since the gas-detection principle is a physical process (not a chemical reaction), and therefore, poisoning of the sensor is not an issue (although dirt/contamination on the optics needs to be considered in the sensor design).

Regarding the light source for these optical techniques, it is worth noticing that stable single-longitudinal-mode (SLM) erbium-doped fiber lasers (EDFLs) have attracted great interest recently, because they can be potentially applied in fiber sensing systems, dense wavelength division multiplexed (DWDM) communications, high-resolution spectroscopy, and microwave photonics fields [15]. Several approaches have been employed to implement SLM operation in EDFLs, such as the use of saturable absorbers [16], short linear cavity distributed Bragg reflector fiber lasers, fiber distributed feedback lasers, and fiber ring lasers [17] with different ring-cavity configura- 


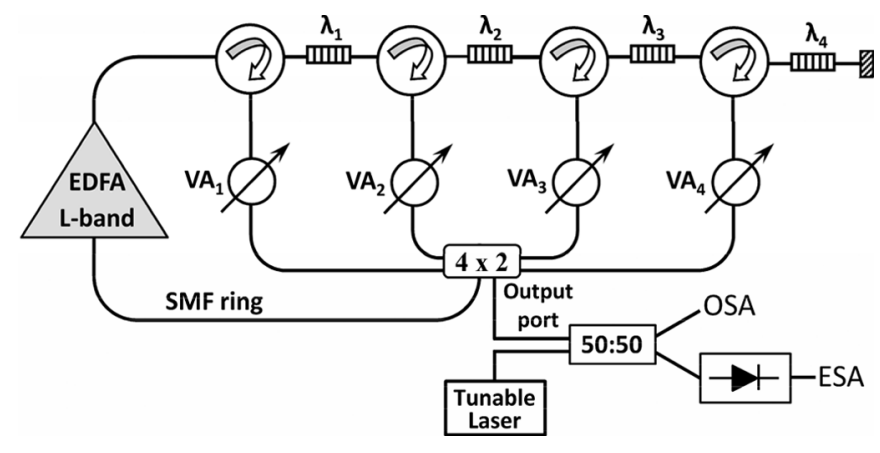

Fig. 1. Proposed single-longitudinal-mode four-wavelength fiber laser for sensors applications.

tions. However, the fiber ring laser unavoidably generates an enormous number of densely spaced longitudinal modes lying beneath the erbium gain profile due to the required intracavity optical components, connecting fibers, and a rather long cavity length. Thus, fiber ring lasers usually perform unstably with a larger linewidth due to the multimode oscillation, mode competition, and hopping. As mentioned above, to achieve SLM operation, a number of techniques have been proposed to ensure a long-term stable performance in EDFLs. Several previous studies proposed by the authors [17] show that, a single-mode operation of these laser systems in the C-band can be achieved when we emit simultaneously two wavelengths using a special ring cavity configuration. In this paper, we report, for the first time to our knowledge, a single-mode operation of an EDFL system operating in the L-band specially designed for sensing applications; when we emit simultaneously four wavelengths using a specific ring cavity configuration. The stable SLM operation in this band is also guaranteed if the output power of all channels is similar. This implies that it is possible to avoid the utilization of additional optical filtering techniques (that reduce the optical efficiency) to achieve the SLM operation.

\section{EXPERIMENTAL SETUP}

The experimental setup of the proposed multiwavelength erbium-doped fiber ring laser (MEDFRL) is shown in Fig. 1. This setup comprises an L-band EDFA module (EDFA-B-L-33 provided by MANLIGHT), four circulators, four FBGs in the L-band, four variable attenuators (VA) and one $4 \times 2$ optical coupler. This coupler is used for both collecting the four arms signals and extracting part of the optical output laser from the ring to the output port. The wavelength selection is carried out by means of FBGs and are centered at $1587.7 \mathrm{~nm}, 1591.9 \mathrm{~nm}$, $1599.9 \mathrm{~nm}$ and $1610.2 \mathrm{~nm}$ with a corresponding full-width at half maximum (FWHM) of $0.17 \mathrm{~nm}, 0.18 \mathrm{~nm}, 0.27 \mathrm{~nm}$ and 0.08 $\mathrm{nm}$, respectively. In this topology, the FBGs reflected signals are inserted into the ring by using optical circulators conforming a serial topology and ensuring unidirectional operation and therefore, avoiding the spatial hole-burning (SHB) effect. Because of this, the use of isolators to ensure the unidirectional oscillation in this configuration was not necessary.

One of the major problems in multiwavelength ring lasers is that the oscillation threshold power for each wavelength is different due to the non-uniform shape of the EDF gain profile. As

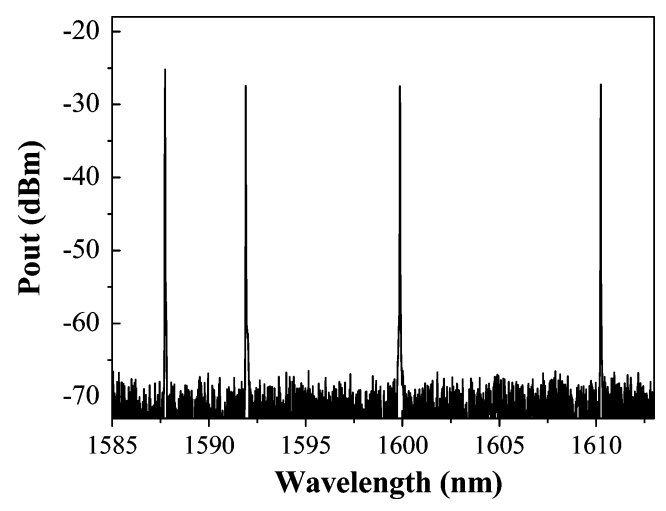

Fig. 2. Output optical spectrum for the MEDFRL circulators configuration with a pump power of $23 \mathrm{dBm}$.

a consequence, variable attenuators (VA) have been connected to each FBG in order to correctly adjust the cavity losses on each wavelength to achieve oscillation of the system in all the desired channels. All the free terminations have been immersed in refractive-index-matching gel to avoid undesired reflections. Several previous studies show that, in order to simplify this kind of setups, polarization maintaining (PM) components can be eliminated because of not having significant impact on the output power stability [18]. The laser output is monitored by an optical spectrum analyzer (OSA) with a highest spectral resolution of $0.01 \mathrm{~nm}$. Single-longitudinal-mode lasing is verified by heterodyne detection of the output signal. Each laser line is combined with the output of a commercial tunable laser source (TLS) with a linewidth of $100 \mathrm{KHz}$ closely located in the spectrum. In this way, the beating signal is observed by means of an ESA, whose resolution bandwidth can be as good as $1 \mathrm{~Hz}$.

\section{EXPERIMENTAL RESULTS AND DISCUSSIONS}

The output spectrum of the MEDFRL for a $23 \mathrm{dBm}$ pump power is illustrated in Fig. 2. As can be shown in this figure four lasing channels are obtained. The power of each of the four output channels is around $-27 \mathrm{dBm}$. For the four channels, the signal power is more than $50 \mathrm{~dB}$ higher than the amplified spontaneous emission (ASE) noise floor. Several previous studies confirm that these values are reasonably good for most sensor applications [19].

As it is well known, in this type of configurations, multiple longitudinal modes are supported by the cavity. However, as it will be exposed in this work, under some specific conditions, such as for similar pumping levels, a single-mode operation of the laser can be achieved. As reported in previous works by the authors [17], a SLM fiber ring laser can be made to annihilate the mode competition by using multiple lasing wavelengths. In this work, owing to the interaction of the seed light produced from one channel to the others and vice versa, multiple-longitudinal-mode oscillation can be suppressed, as can be seen in Fig. 3, and thus the mode competition and mode hopping is not produced. In these figures, the beat note spectrum between the tunable laser and the obtained single-longitudinal mode signal is presented. They show the obtained results when this tunable laser is close tuned to the first (a), second (b), third (c), and 

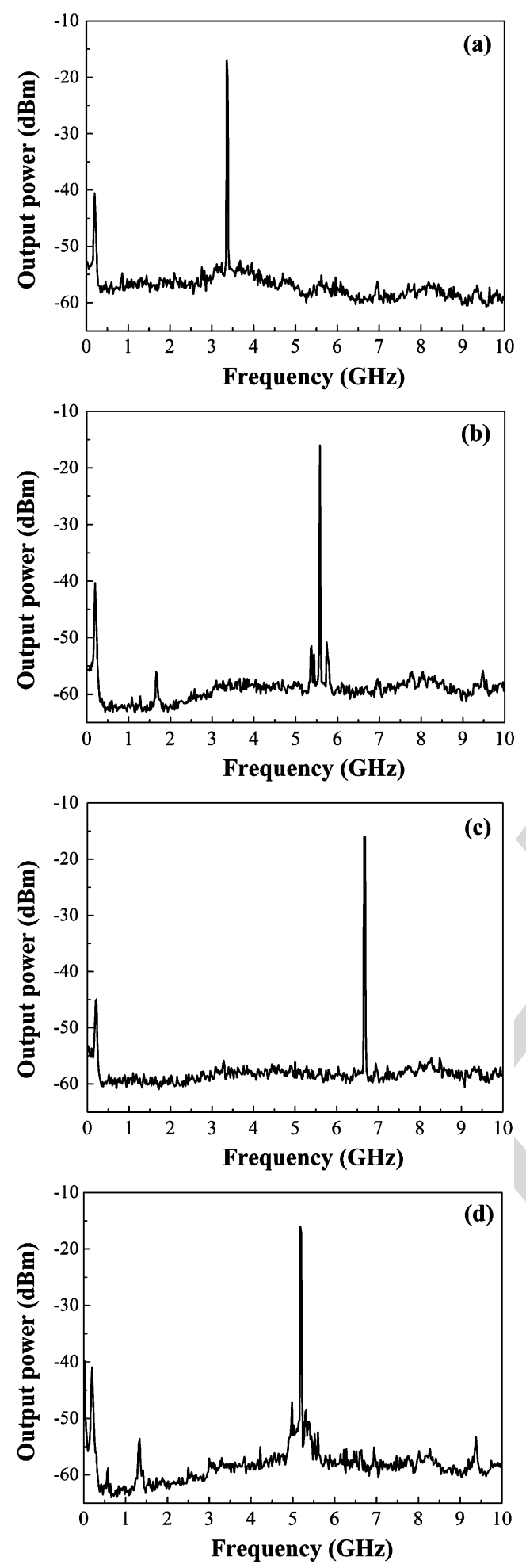

Fig. 3. Output optical spectrum measured by the ESA for the MEDFRL circulators configuration when the tunable laser was tuned to the first (a), second (b), third (c) and fourth (d) wavelength laser emission.

fourth (d) wavelength laser emission in order to perform heterodyne detection.

In addition to this, authors would like to underline the fact that there are no peaks at low frequency except for a beat that is attributed to the residual side modes of the TLS. Thus, we can

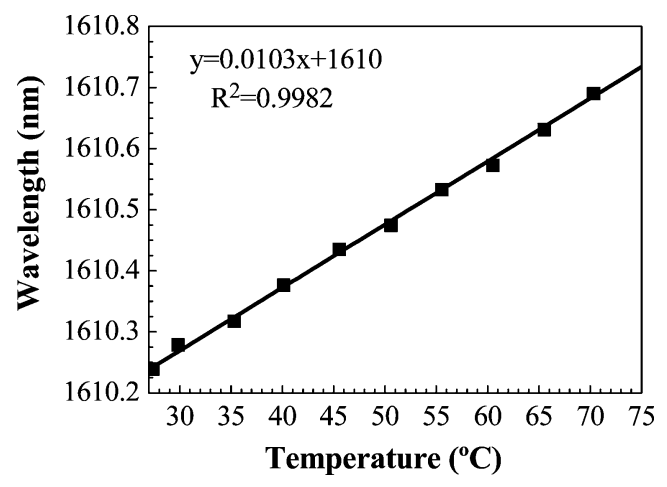

Fig. 4. Wavelength shift of one sensor against temperature for the three-wavelength EDFRL.

assure that these four wavelength lasers have a single-longitudinal mode operation simultaneously. It is also worth noticing that the sidebands of the tunable laser can be also observed, as Fig. 3(b) clearly illustrates, located very close to the beat peak.

Due to the fact that only one peak is obtained for all the studied cases and taking into account that the resolution bandwidth of the ESA is good enough to discriminate two longitudinal modes separated about $8 * 10^{-12} \mathrm{~nm}$, we can assure that this laser presents a SLM operation condition for all the channels at the same time. These measurements have been repeated at different emission wavelengths in the L-band. In all cases, an SLM operation for all the channels is achieved when all of these lasing wavelengths are oscillating simultaneously with similar output powers by using the VAs to adjust the cavity losses.

The utilization of this new laser for sensor multiplexing was carried out using the FBGs as temperature sensors. The temperature response of the sensors was tested by heating them on a climatic chamber in the range of $27^{\circ} \mathrm{C}$ to $75^{\circ} \mathrm{C}$. As can be seen in Fig. 4, the center wavelength shift for the laser emission located at $1610 \mathrm{~nm}$ presents a clear linear behavior (the mean square error is very close to 1 ) and a temperature sensitivity of about $10 \mathrm{pm} /{ }^{\circ} \mathrm{C}$ was measured for a three-wavelength EDFRL.

On the other hand, the center wavelength shift for the other wavelengths at room temperature was about $0.02 \mathrm{~nm}$ and 0.06 $\mathrm{nm}$ for the FBGs centered at 1587.7 and $1591.1 \mathrm{~nm}$ respectively. Due to this constant value, these two lasers at constant temperature could be use as reference measurements. Next two figures (Fig. 5(a) and (b)) show the obtained results.

The laser output power can suffer some changes with time. For this reason, the temporal variations of the output power were also measured and analyzed on this MEDFRL. The instability is defined as the output power for a given interval of time and a specific confidence level (CL). This is the probability value associated with a confidence interval (CI), given as a percentage. The $\mathrm{CI}$ is the estimated range of values where the parameter of interest is included [20].

It has been also experimentally demonstrated that when a SLM behavior is achieved, lower output power fluctuations are obtained. We have tested the sensor system during a period of 30 minutes both for the SLM and MLM operation and a CL of $90 \%$ was considered.

As mentioned above, a SLM operation can be obtained when all the lasing wavelengths are oscillating simultaneously with 

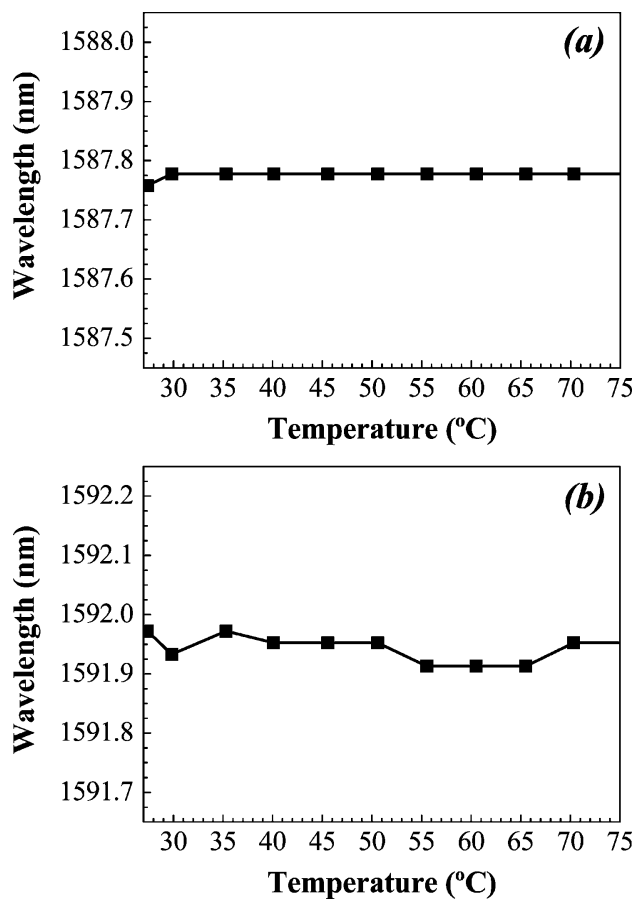

Fig. 5. Wavelength shift of the FBGs at room temperature centered at 1587.7 $\mathrm{nm}$ (a) and $1591.1 \mathrm{~nm}$ (b) respectively for the three-wavelength EDFRL.

similar output powers [17]. In order to demonstrate this stability improvement, two different analyses were carried out. For the first one, both lasing wavelengths were selected to have similar output power levels (about $-26 \mathrm{dBm}$ ) so, a SLM was obtained. For the second one, this condition was modified; by using the VAs to adjust the cavity losses, both wavelength peaks were oscillating with different output power levels, obtaining a MLM behavior. In this study we focused on the wavelength with the highest output power level (about $-24 \mathrm{dBm}$ ), that is, the one that oscillates at $1610 \mathrm{~nm}$.

The obtained results from these analyses can be seen in Fig. 6 . For a dual-wavelength EDFRL at room temperature, the power averaged variation was around $2.1 \mathrm{~dB}$ for a multiple-longitudinal mode (MLM) behavior, and this value improves to be about $1.2 \mathrm{~dB}$ for a SLM operation, even when the analyzed wavelength had a higher output power level, that is suppose to have a better stability.

Although we can conclude that a SLM operation implies also a remarkable improvement in terms of output power variations, the obtained results show that this configuration requires a more accurate control of the cavity losses on each wavelength to achieve oscillation of the system in all the desired channels and in order to obtain a very good equalized lasing system. This is an important study that we plan to perform because, in practice, it is not straightforward to achieve the optimum condition because of the availability of the optical variable attenuators and, therefore, it is relevant to know the level of performance degradation when, for example, the output power levels have great different values.

\section{CONCLUSION}

A single-longitudinal-mode four-wavelength laser configuration for sensing applications in L-band has been experimen-

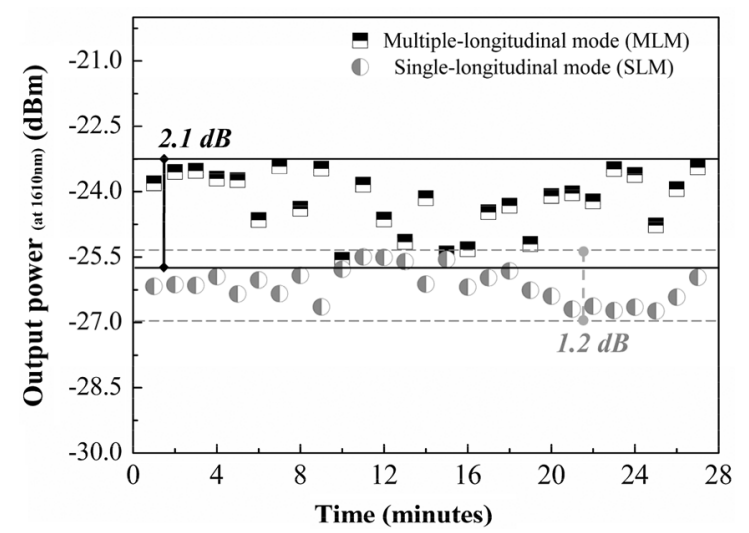

Fig. 6. Fluctuations of peak power versus time generated by a dual-wavelength EDFRL at room temperature for a multiple-longitudinal mode (MLM) and a single-longitudinal mode (SLM) behavior.

tally demonstrated in this work. The advantage of extending the range of wavelength operation of fiber lasers into the L-band expands the limits of present day tunable sources and hence the detection of gases with absorption lines in the L-band region. The fiber structure presented here is based on ring resonators, and employs fiber Bragg gratings to select the operation wavelengths. The stable SLM operation is guaranteed when all the lasing channels present similar output powers. Operation of the laser structure for temperature sensing was shown. It was also experimentally demonstrated that when a SLM behavior is achieved, lower output power fluctuations are obtained.

\section{REFERENCES}

[1] [Ssue number and month?] G. A. Ball, W. W. Morey, and P. K. Cheo, "Single- and multipoint fiber-laser sensors," IEEE Photon. Technol. Lett., vol. 5, pp. 267-270, 1993.

[2] [ssue number and month?] Jarabo, S. Abad, and M. López-Amo, "New WDM amplified network for optical sensor multiplexing," IEEE Photon. Technol. Lett., vol. 11, pp. 1644-1646, 1999

[3] [Issue number and month?]P. Peng, H. Tseng, and S. Chi, "Long-distance FBG sensor system using a linear-cavity fiber Raman laser scheme," IEEE Photonics Technol Lett., vol. 16, pp. 575-577, 2004.

[4] Y. Han, T. V. A. Tran, S. Kim, and S. B. Lee, "Multiwavelength Raman-fiber-laser-based long-distance remote sensor for simultaneous measurement of strain and temperature," Opt. Lett., vol. 30, pp. 1282-1284, 2005

[5] Y.Han, "Long-distance remote sensors for simultaneous measurement of strain and temperature based on multiwavelength fiber lasers," in Proc. SPIE, 2009, art. no. 75032B.

[6] M. Fernandez-Vallejo, S. Díaz, R. A. Perez-Herrera, D. Passaro, S. Selleri, M. A. Quintela, J. M. López-Higuera, and M. Lopez-Amo, "Resilient long-distance sensor system using a multiwavelength Raman laser," Meas. Sci. Technol., vol. 21, p. 5, 2010, 094017.

[7] H. Chen, M. Leblanc, and G. W. Schinn, "Gain enhanced L-band optical fiber amplifiers and tunable fiber lasers with erbium-doped fibers," Opt. Commun., vol. 216, no. 1-3, pp. 119-125, 2003.

[8] A. Bellemare, M. Karasek, C. Riviere, F. Babin, G. He, V. Roy, and G. W. Schinn, "A broadly tunable erbium-doped fiber ring laser: Experimentation and modeling," IEEE J. Sel. Topics Quantum Electron., vol. 7, no. 1, pp. 22-29, Jan./Feb. 2001.

[9] S. Yamashita and M. Nishihara, "L-band erbium-doped fiber amplifier incorporating an inline fiber grating laser," IEEE J. Sel. Topics Quantum Electron., vol. 7, no. 1, pp. 44-48, Jan./Feb. 2001.

[10] G. Stewart, K. Atherton, H. B. Yu, and B. Culshaw, "An investigation of an optical fibre amplifier loop for intra-cavity and ring-down cavity loss measurements," Meas. Sci. Technol., vol. 12, no. 7, pp. 843-849, 2001. 
[11] J. Marshall, G. Stewart, and G. Whitenett, "Design of a tunable L-band multi-wavelength laser system for application to gas spectroscopy," Meas. Sci. Technol., vol. 17, no. 5, pp. 1023-1031, 2006.

[12] C. Massie, G. Stewart, G. McGregor, and J. R. Gilchrist, "Design of a portable optical sensor for methane gas detection," Sens. Actuators, B: Chem., vol. 113, pp. 830-836, 2006.

[13] A. M. Cubillas, M. Silva-Lopez, J. M. Lazaro, O. M. Conde, M. N. Petrovich, and J. M. Lopez-Higuera, "Methane detection at 1670-nm band using a hollow-core photonic bandgap fiber and a multiline algorithm," Opt. Exp., vol. 15, pp. 17570-17576, 2007.

[14] [Please provide more information] $\mathrm{P} . \mathrm{T}$. Mosely, J. O. W. Norris, and D. E. Williams, Techniques and Mechanisms in Gas Sensing 1991

[15] J. Sun and L. Huang, "Single-longitudinal-mode fiber ring laser using internal lasing injection and self-injection feedback," Opt. Eng., vol. 46, p. 6, 2007, art. no. 074201.

[16] M. Horowitz, R. Daisy, B. Fischer, and J. Zyskind, "Narrow-linewidth, singlemode erbium-doped fibre laser with intracavity wave mixing in saturable absorber," Electron. Lett., vol. 30, pp. 648-649, 1994.

[17] [Ssue number and month?]. A. Perez-Herrera, I. Canales, M. Fernández-Vallejo, M. Lopez-Amo, and J. M. López-Higuera, "Stabilization of dual-wavelength erbium-doped fiber ring lasers by single-mode operation," IEEE Photon. Technol. Lett., vol. 22, pp. 368-370, 2010.

[18] R. A. Pérez-Herrera, M. A. Quintela, M. Fernández-Vallejo, A. Quintela, M. López-Amo, and J. M. López-Higuera, "Stability comparison of two ring resonator structures for multiwavelength fiber lasers using highly doped er-fibers," J. Lightw. Technol., vol. 27, pp. 2563-2569, 2009.

[19] S. Diaz, S. Abad, and M. Lopez-Amo, "Fiber-optic sensor active networking with distributed erbium-doped fiber and Raman amplification," Laser Photon. Rev., vol. 2, pp. 480-497, 2008.

[20] [Online]. Available: http://www.stats.gla.ac.uk/steps/glossary/confidence_intervals.html

Rosa Ana Perez-Herrera was born in Cantabria, Spain, in February 1979. She received the telecommunications engineering degree from the University of Cantabria, Spain, in 2004. In 2010 she received the Ph.D. degree from the Public University of Navarra, Navarra, Spain.

In 2005 she joined the Optical Communications Group at the Department of Electrical and Electronic Engineering of the Public University of Navarra (Pamplona, Spain). During this period, she was a visiting Ph.D. student at INESC in Unidade De Optoelectronica E Sistemas Electronicos in Porto (Portugal), at the School of Engineering and Mathematical Sciences at City University of London and at the Department of Information Engineering at Parma University (Italy) among others. In 2009, she became an Assistant Professor in the Electrical and Electronic Engineering department of the Public University of Navarra. Her research interests are in Raman amplifiers, erbium-doped amplifiers, fiber-optic sensors and multiplexing architectures.

Ángel Ullán Nieto was born in Santander, Spain, in 1979. In 2004 he graduated in physics at the University of Zaragoza, Spain. In 2007 he joined the Photonics Engineering Group at the University of Cantabria, Spain, where he is currently pursuing the Ph.D. degree

He joined the Liquid Crystals and Photonics Group at Ghent University, Belgium, where he was doing research in the field of Organic LEDs for two years. His research is focused on Brillouin scattering for fiber sensors and Brillouin fiber lasers.

Daniel Leandro was born in Navarra, Spain, in November 1984. He received Telecommunication engineering degree in 2010 from the Public University of Navarra, Spain.

His research interests include Raman and erbium-doped amplifiers, fiber Bragg grating sensor and multiplexing architectures.

Montserrat Fernández-Vallejo was born in Navarra, Spain, in February 1983. She received the M.Sc. degree in telecommunication engineering from the Public University of Navarra, Spain in 2008, where she is currently working toward the Ph.D. degree.
She was a visiting Ph.D. student at the department of information engineering at Parma University, Italy, and at the Institute of Photonic Technology IPHT, Germany. Her research is focused on Raman amplifiers, erbium-doped amplifiers, fiber-optic sensors and multiplexing architectures.

María Angeles Quintela was born in Santander, Spain, in 1974. She received both her Telecommunication Technical Engineering degree and her Telecommunication Engineering degree at the University of Cantabria, Spain, where she received the Ph.D. degree in 2005.

In 1998, she joined the University of Cantabria as a lecturer. Her teaching topics include electronic components and optical communications. Her main research is on computer modelling and experimental characterisation of fiber lasers. She has co-authored more than 60 papers, presented in conferences and scientific journals.

Alayn Loayssa was born in San Sebastian, Spain, in 1973. He holds a M.Sc. degree in telecommunication engineering in 1997 and the Ph.D. degree in optical communications in 2001, both from the Public University of Navarra, Spain.

In 2001 he was visiting Research Fellow at the University of Melbourne, Australia. Currently, he is an Associate Professor at the Electrical and Electronic Engineering Department of the Public University of Navarra and a member of the Optical Communications Research group, where he leads research on microwave photonics, optical measurements applications and Brillouin distributed sensing.

Dr. Loayssa has served as member of the Technical Program Committee of the IEEE International Microwave Photonics Conference (MWP). In addition, he is a member of the editorial board of the International Journal of Microwave Science and Technology.

José Miguel López-Higuera was born in February 1954, in the village of Ramales de la Victoria, Cantabria (Spain). He received the Telecommunication Technical Engineering degree in the Universidad Laboral de Alcalá de Henares, and the Telecommunication Engineering degree from the Universidad Politécnica de Madrid (UPM), Spain. He received the Ph.D. degree in telecommunication engineering, with an extraordinary award, in UPM.

He founded and is the head of the Photonics Engineering Group of the TEISA Department in the University of Cantabria. Presently, he works in the development of Photonics Instrumentation, photonic/optical fibre sensor systems for civil engineering, electrical power, environmental and smart structures and for optical diagnostics for a wide range of applications. He has directed more than fifty R\&D projects and has written or co-written more than four hundred publications in the form of books, chapters of books, papers and conferences, both national and international, and obtained 10 industrial publications (patents). $\mathrm{He}$ is the editor and coauthor of several books, including Optical Sensors (UC, 1998), the "Handbook of Photonic Sensing Technology (Wiley, 2002), and he is the co-editor of the book Engineering a High-Tech Business: Entrepreneurial experiences and Insights (SPIE, 2008).

Prof. López-Higuera is a member of the SPIE and OSA.

Manuel Lopez-Amo (M'91-SM'98) was born in Madrid, Spain, in 1960. He received the M.Sc. degree in telecommunication engineering and the Ph.D. degrees from the Universidad Politécnica de Madrid, Spain in 1985 and 1989, respectively.

From 1985 to 1996, he belonged to the Photonic Technology Department of the Universidad Politécnica de Madrid, where in 1990 he became an associate professor. In 1996, he moved to the Universidad Pública de Navarra, Pamplona, Spain, where he became a Full Professor in the electrical and electronic engineering department and he is currently the head of the optical communications group of this department. He has been Chairman of the Optoelectronic Committee of Spain. He has been leader of more than 30 research projects and he has coauthored more than 150 works in international refereed journals and conferences related with fiber-optic networks, optical amplifiers, fiber-optic sensors, and integrated optics.

Prof. Lopez-Amo is a member of the technical committees of the International Conference on fiber optic sensors (OFS), the European Workshop on optical fiber sensors (EWOFS), and European cost TD1001 action, among others. His a member of the OSA. 